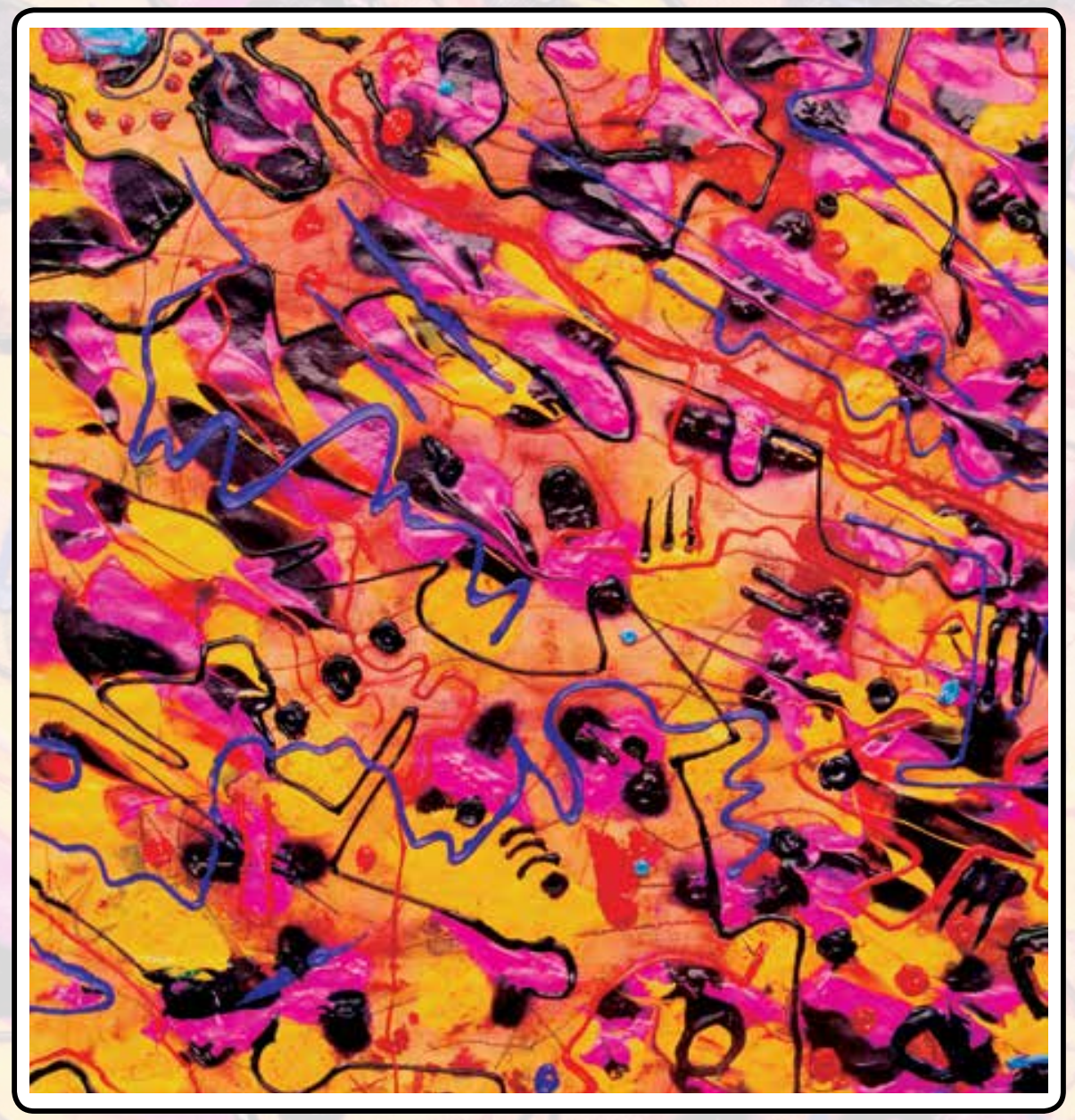

Camilo Umaña V.

Sin título

Acrílico sobre Lienzo 100 x 80 



\title{
MODELOS PARA LA IDENTIFICACIÓN DE STAKEHOLDERS Y SU APLICACIÓN A LA GESTIÓN DE LOS PEQUEÑOS ABASTECIMIENTOS COMUNITARIOS DE AGUA
}

\author{
MODELS FOR THE IDENTIFICATION OF STAKEHOLDERS AND THEIR \\ APPLICATION IN THE MANAGEMENT OF SMALL COMMUNITY WATER \\ SUPPLIES
}

Andrea Bernal ${ }^{1}$ y Luis Arturo Rivas ${ }^{2}$

\begin{abstract}
Resumen
Este es un trabajo de revisión sobre la teoría de stakeholders (actores, agentes o grupos de interés). El análisis de stakeholders resulta útil para la rendición de cuentas, la definición de la propuesta de creación de valor, el diseño de la estrategia, el desarrollo de las operaciones y en el fortalecimiento de la gobernabilidad, sin embargo, su operacionalización resulta difusa. Este trabajo describe críticamente los modelos clásicos y aplicados para identificar a los stakeholders, y formula recomendaciones para el mapeo de stakeholders especialmente en pequeñas organizaciones y como resultado del análisis se presenta un modelo para el mapa de actores de pequeños abastos de agua ${ }^{3}$
\end{abstract}

\section{Palabras Clave}

Stakeholders, grupos de interés, pequeños abastos de agua

\section{Clasificación JEL: M19}

\begin{abstract}
This is a review paper on the theory of stakeholders (actors, agents or stakeholders). The stakeholder analysis is useful for accountability, the proposed definition of value creation, design strategy, operations development and strengthening of governance, however their operation, are blurred. This paper describes the classic models and critically applied to identify stakeholders, and make recommendations for mapping stakeholders especially in small organizations and as a result we present a model for the young actor map of water supplies
\end{abstract}

\section{Keywords}

Stakeholders, interest groups, small water supplies

1 Maestra en Dirección y Gerencia de Empresas, Candidata a Doctora en Ciencias Administrativas, Escuela Superior de Administración (ESCA) Santo Tomás del Instituto Politécnico Nacional de México (IPN). Ha sido docente de la Universidad del Rosario en Bogotá - Colombia y Consultora del Banco Interamericano de Desarrollo (BID) en temas de abastecimiento rural de agua y saneamiento. Actualmente realiza estudios en desarrollo sostenible en la Universidad de Exeter - Reino Unido. Correo electrónico: artenoble@hotmail.com.

2 Doctor en Ciencias Administrativas y Doctor (c). en Estudios Europeos. Catedrático de la ESCA Santo Tomás del Instituto Politécnico Nacional de México - IPN. Investigador Nacional. Correo electrónico: larivas33@ hotmail.com

3 La denominación de pequeños abastos comunitarios de agua proviene de la traducción al español del apelativo "Small Community Water Supplies" o (SCWS) por sus siglas en inglés, empleado por la Organización Mundial de la Salud en la guía para la formulación de planes de seguridad del agua, aún no traducida al español. (WHO, 2012) 


\section{Introducción}

Dado el interés que despierta la teoría de stakeholders como nuevo paradigma de la administración, este documento describe la evolución de la teoría de stakeholders, de los referentes normativos contenidos en las guías para el reporte de sostenibilidad GRI G 3.1. y Account Ability AA1000 SES, (ver numeral 4), de los modelos clásicos para la identificación de actores o grupos de interés, así como un análisis crítico de dichos modelos como alternativas de operacionalización de la teoría. Por último, se presenta un modelo de mapa de actores aplicado a la gestión de pequeños abastos de agua, formulado como resultado de la investigación en mención (Bernal \& Rivas, 2012).

\section{Evolución de la teoría de los stakeholders}

A partir del trabajo seminal de Freeman (1984) la noción de "stakeholders" traducido al español como "grupos de interés", "actores" o "agentes" - empezó a ser ampliamente integrada a la estrategia de las organizaciones. Sin embargo, el concepto ha sido revisado desde diversas perspectivas teóricas y ha alcanzado no menos de treinta y ocho definiciones distintas (Mitchel, Agle \& Woods, 1997). A su vez, el trabajo de Freeman (1984) ha sido revisado y actualizado para incluir los avances conceptuales alcanzados durante más de tres décadas (Freeman, Harrison \& Wicks, 2008) (Freeman- Harrison- Wicks- Parmar \& De Colle, S 2010).

Vale ilustrar textualmente la definición de grupos de interés contenida en la guía G3.1 del GRI (2011) para el término inglés "stakeholders":

"Grupos de interés: Se entiende por grupos de interés aquellos grupos o particulares: (a) que pueda esperarse, dentro de lo razonable, sean afectados de manera significativa las actividades, productos $\mathrm{y} / \mathrm{o}$ servicios de la organización; o (b) cuyas acciones pueden esperarse, dentro de lo razonable, que afecten a la capacidad de la organización para implantar con éxito sus estrategias y alcanzar sus objetivos" (GRI, 2011, p.67).

Varios autores han enunciado algunas categorías mínimas para la identificación de stakeholders, como los ejemplos que se citan a continuación: 
Ilustración 1

Stakeholders internos y externos

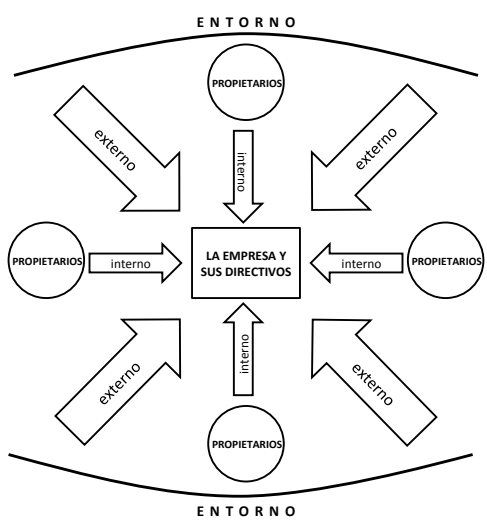

Fuente: Freeman 1984: p. 17

\section{Ilustración 2}

Stakeholders primarios y secundarios

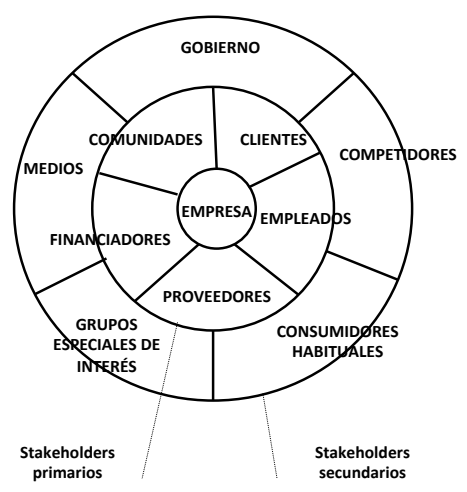

Fuente: Freeman, Harrison \& Wicks, 2008, p. 7

Ilustración 4

Campo de juego para la eco-ventaja

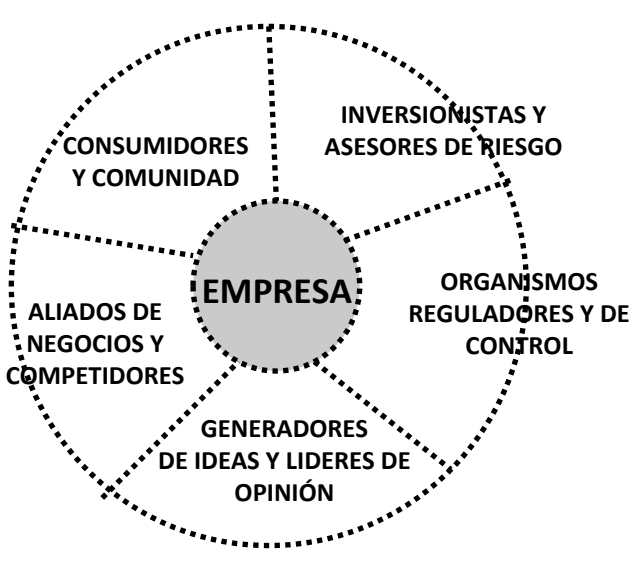


Las ilustraciones 1, 2, 3 y 4, permiten apreciar el debate sobre sí el medio ambiente debería ser incluido como una categoría autónoma o no. A partir de la definición de grupos de interés (GRI, 2011) que no dista del concepto de stakeholders inicialmente acuñado por Freeman (1984), se infiere que solamente los individuos o grupos que gocen de los atributos de la personalidad (capacidad y voluntad) pueden ser stakeholders, pues ello implica la posibilidad de toma de decisiones por seres humanos. Así, la identificación e inclusión de stakeholders en la estrategia empresarial radica en la interacción con personas o grupos reconocidos como titulares o representantes de un interés. Por otra parte, y desde un enfoque no antropocéntrico, se invoca al medio ambiente como un stakeholder con voz propia, no necesariamente representado por algún individuo o grupo de interés, ello con el fin de que las preocupaciones por la sostenibilidad se inserten efectivamente en la estrategia de la empresa (Driscoll \& Starik, 2004)

Otro debate surge a partir de la clasificación de stakeholders, que oscila entre diversas dialécticas (González, 2001): stakeholders internos y externos de acuerdo con los límites físicos de la organización y su relación con el entorno (Freeman, 1984); stakeholders directos o indirectos (o sociales y no-sociales) según su tipo de relación con la organización y la capacidad que tienen de representarse a sí mismos o por interpuesta persona (Wheeler \& Sillanpaa, 1997); y stakeholders primarios y secundarios según la necesidad del stakeholder para la supervivencia de la empresa (Freeman et al, 2008). Si bien estas clasificaciones tienen como finalidad la delimitación de las categorías de stakeholders para su operacionalización, la experiencia demuestra cómo la complejidad de las relaciones entre stakeholders es inasible desde estas clasificaciones, y en un mundo cada vez más interconectado, las barreras se hacen difusas lo que resta utilidad a estas restricciones teóricas; las empresas hoy temen más a los reclamos de los grupos de consumidores conectados en Internet o a las reacciones de los medios, que a los requerimientos de sus empleados o sus accionistas (Esty \& Winston, 2011). En todo caso, el reto operativo radica en la segmentación de cada una de las categorías; la identificación de stakeholders sólo es completa cuando los stakeholders están representados con su propia voz en los escenarios de participación.

Donaldson y Preston(1995) iniciaron el debate sobre el problema de la justificación, al postular que la teoría de stakeholders es, a su vez, descriptiva e instrumental, pero que alcanza su fin ulterior al desplegarse como una teoría normativa. Esta discusión inspira una pregunta: ¿Por qué la teoría de los stakeholders debe ser preferida frente a otras alternativas? Al revisar los enfoques teóricos enunciados, vale profundizar en esta reflexión.

Freeman et al. (2010) en la actualización de su trabajo académico, aclaran lo siguiente: 
"La teoría de los Stakeholders es fundamentalmente una teoría acerca de cómo el negocio trabaja mejor, y como podría trabajar. La teoría de los stakeholders es sobre la creación de valor y transacciones y cómo manejar un negocio efectivamente. Lo "efectivo" puede ser visto como "crear tanto valor como sea posible". Si la teoría de los stakeholders es para resolver problemas de creación de valor y transacciones, debe demostrar cómo los negocios pueden de hecho ser descritos a través de relaciones entre stakeholders. Si es para resolver el problema de la ética del capitalismo, debe mostrar cómo un negocio puede ser manejado para rendir cuentas de sus efectos y responsabilidades a todos los stakeholders. Y si es para resolver el problema de la mentalidad administrativa, debe adoptar una manera práctica de poner juntos el negocio y la ética que sea implementable en el mundo real". (Freeman et al, 2010, p. 17)

Kochan y Rubinstein (2000) exploran la teoría de stakeholders desde una perspectiva comunicacional, de riesgo compartido en el que cada quien entrega y recibe algo a cambio. En sus palabras:

"En suma, para que una empresa funcione exitosamente, se requiere movilizar esfuerzos discrecionales ente los empleados, se necesita altos niveles de comunicación a través de los grupos y sus funciones, y los conflictos deben ser expuestos y resueltos efectivamente. Dados los múltiples intereses del reparto de poder, la resolución de conflictos debe ser tenida como una función especialmente crítica en organizaciones de stakeholders" Kochan \& Rubinstein, 2011, p. 378)

De acuerdo con Sachs y Ruhli (2011), la teoría de los stakeholders es un nuevo paradigma de la estrategia organizacional llamado a sacudir los fundamentos de la teoría de la firma y el capitalismo, critica la racionalidad limitada del desempeño económico y anota que la cooperación es prioritaria y que la competición es una propiedad emergente, no un elemento básico del capitalismo. Así, según estos autores, la creación de valor depende de un balance entre la cooperación y la competencia entre redes de stakeholders. En sus términos:

"Los negocios en el siglo XXI deben ser vistos como una institución que crea valor para los clientes, proveedores, empleados, comunidades, financiadores y la sociedad (...) en efecto el nuevo contrato social contiene tres licencias: (1) licencia para operar (2) licencia para innovar y (3) licencia para competir. La combinación conduce a una nueva agenda para pensar acerca del propósito de la firma, su visión estratégica y su modelo de negocios para la creación de valor" (Sachs \& Ruhli, 2011, p. 69)

Por otra parte, Donaldson y Preston. argumentan que la teoría de stakeholders es en esencia, normativa, en cuanto:

“(...) La última implicación administrativa de la teoría de stakeholders es que los directivos deben reconocer la validez de los diversos intereses de los stakeholders, y deberían intentar responder a ellos dentro de una estructura de mutuo apoyo porque éste es un requerimiento moral para la legitimación de la función administrativa" (Donaldson \& Preston, 1995, p. 87). 
Así, la teoría de stakeholders se identifica con el pensamiento neoinstitucional, que parte del supuesto de que los grupos humanos crean instituciones (reglas de juego) para reducir el grado de incertidumbre de sus interacciones. Estas instituciones pueden ser más o menos formales, pero son reconocidas como tales una vez que el grupo social confía en ellas y se ciñe a su estructura. Así mismo, el neoinstitucionalismo busca comprender cómo funcionan los arreglos institucionales, en especial, por la manera en que los stakeholders realizan sus transacciones. En palabras de Falcao y Fontes (1999):

"La perspectiva normativa se basa en una visión institucional de la organización, definida como un escenario de múltiples intereses competitivos, y a veces conflictivos, en un espacio social en el que los stakeholders juegan en diferentes posiciones de poder, y en el cual la sustentabilidad organizacional depende de pactos (trade-offs) y arreglos cooperativos específicos (principalmente entre stakeholders primarios, sin los cuales la organización sucumbe, y otros momentáneamente relevantes" (Falcao \& Fontes, 1999, p.2).

Si la teoría de stakeholders conduce a una representación de los arreglos institucionales existentes, al preguntarse cómo se llegó a ellos, se cuenta con un instrumento de gobernanza que permite ilustrar las relaciones de influencia/poder en un contexto dado:

"La construcción de relaciones institucionales se describe en función del contexto temporal y espacial, pero también está condicionada por las percepciones de los actores sobre las reglas de juego que, a su vez, surgen de las referencias culturales, las preferencias, las identidades, la historia y la naturaleza de cada actor implicado. La pluralidad de reglas de juego y su interpretación entrañan negociaciones y fricciones que harán evolucionar permanentemente a las instituciones, y así, las reglas e instituciones emergentes evolucionan en función de los conflictos, de las demostraciones de fuerza y de los compromisos según visiones y representaciones del mundo. De esta manera, el neoinstitucionalismo contribuye a entender el rol de los procesos sociales y políticos en el surgimiento de compromisos institucionales." (Barrau, 2010. p. 14)

Las premisas anteriores dan cuenta de la evolución de la teoría de stakeholders, como ejercicio multidisciplinar que tiene mucho que aportar en el nuevo entendimiento de las organizaciones como instituciones claves para el desarrollo social desde múltiples escenarios, como son la rendición de cuentas, la creación de valor, la estrategia organizacional, la resolución de conflictos y el buen gobierno, entre otros. Si bien las razones para abordar el enfoque de stakeholders son suficientes y motivadoras, esta teoría requiere ser operacionalizada a través de referentes, métodos y representaciones generalmente aceptadas, para su amplia implementación. 


\section{Desafíos en la construcción de modelos para la identificación de stakeholders}

En primer lugar, frente a la pregunta de: ¿Cuál es la información necesaria para analizar los stakeholders? es necesario contar con: (1) referentes normativos para delimitar los contenidos del modelo; (2) con modelos para procesar la información y evaluar los resultados, que cuenten con métodos estándar de valoración de los datos y representaciones textuales o gráficas de los resultados. Todos estos elementos deben ser congruentes entre sí.

En segundo lugar, cabe preguntarse: ¿Cómo se usaría un modelo de análisis de actores? Considerado que los arreglos institucionales son por esencia, procesos de toma de decisiones, la intención es que el modelo ayude a tomar decisiones más rápidas y acertadas o sustentar decisiones rutinarias o de política. Así mismo, un modelo debe resultar útil para los académicos que así pueden priorizar su trabajo, promover la investigación y el mejoramiento continuo y generar ideas para el debate, y para los empíricos quienes contarán con herramientas para desplegar su práctica con un sustento más amplio (Pidd, 2010).

A continuación se exploran los referentes normativos y los modelos clásicos para la identificación de stakeholders, y se postulan las restricciones y percepciones sobre dichos modelos, con la intención de derivar elementos de juicio para el posterior desarrollo de un modelo aplicado al análisis de stakeholders de pequeños abastecimientos comunitarios de agua.

Referentes normativos para la identificación de stakeholders Global Reporting Initiative (GRI, 2011) - versión G3.1

La guía para la elaboración de memorias de sostenibilidad de GRI versión G3.1, disponible en español (GRI, 2011), según el principio de participación de grupos de interés, requiere contenidos expresos sobre este tópico, descritos en los numerales 4.14 al 4.17 en los siguientes términos:

"Participación de los grupos de interés: (...) (1) Relación de los grupos de interés que la organización ha incluido; (2) Base para la identificación y selección de grupos de interés con los que la organización se compromete. (Este apartado incluirá el procedimiento de la organización para la definición de sus grupos de interés así como para la determinación de los grupos que participan y los que no); (3) Enfoques adoptados para la inclusión de los grupos de interés, incluidas la frecuencia de su participación por tipos y categoría de grupos de interés.(...), y (4) Principales preocupaciones y aspectos de interés que hayan surgido a través de la participación de los grupos de interés y la forma en la que ha respondido la organización a los mismos en la elaboración de la memoria. (...)" (GRI, 2011: 26).

De esta enumeración se desprende que la inclusión de los grupos de interés surge de un proceso congruente con el enfoque y la cobertura de la memoria de sostenibilidad, 
que puede estar previamente establecido por el marco institucional y legal en el que opera la organización, o en su defecto, por los procesos diseñados específicamente para cada memoria. En todo caso, la identificación de stakeholders según la guía GRI G3.1 (GRI, 2011) no sigue aún un protocolo estándar, y la divulgación de los procesos seguidos para la definición de stakeholders incluidos y su vinculación a la organización es aún potestativa. Por otra parte, la descripción de la participación de grupos de interés no se sujeta al esquema de categorías, aspectos e indicadores que se pueden apreciar en la sección subsiguiente de la guía, para el enfoque de gestión.

\section{Account Ability Series - AA1000SES}

AA1000 es una serie de normas de aseguramiento y estándares de reporte de sostenibilidad de acceso abierto para todo tipo de organizaciones. En atención al principio de inclusividad, fue desarrollada la norma AA1000SES - Stakeholders Engagement Estándar (estándar para el compromiso con los stakeholders), cuya premisa básica es incorporar a los stakeholders en la estrategia, las operaciones y el gobierno de la organización. En la ilustración 5 se presenta la estructura de la AA1000SES y su método para identificar stakeholders:

Ilustración 5. Estructura para el compromiso de los stakeholders
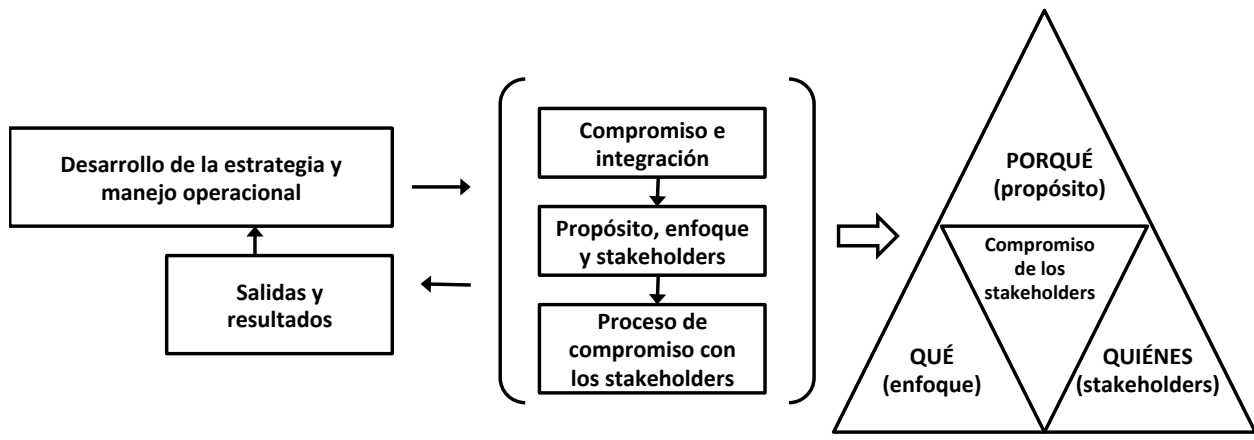
Tabla 1. Atributos para identificar stakeholders según la norma AA1000SES

\begin{tabular}{|l|l|}
\hline Dependencia & $\begin{array}{l}\text { Grupos o individuos que son directa o indirectamente dependientes de las } \\
\text { actividades, productos o servicios de la organización, o de su correlativo } \\
\text { desempeño, o de los cuales la organización es dependiente para operar. }\end{array}$ \\
\hline Responsabilidad & $\begin{array}{l}\text { Grupos o individuos con los cuales la organización tiene o en el futuro puede tener } \\
\text { responsabilidades legales, comerciales, operacionales o ético/morales. }\end{array}$ \\
\hline Tensión & $\begin{array}{l}\text { Grupos o individuos que necesitan atención inmediata desde la organización en } \\
\text { consideración a aspectos financieros, económicos, sociales o ambientales. }\end{array}$ \\
\hline Influencia & $\begin{array}{l}\text { Grupos o individuos que pueden tener impacto en la organización o tomar } \\
\text { decisiones operacionales o estratégicas }\end{array}$ \\
\hline Diversas perspectivas & $\begin{array}{l}\text { Grupos o individuos cuyas diferentes perspectivas pueden llevar a un nuevo } \\
\text { entendimiento de una situación o la identificación de oportunidades, lo que de } \\
\text { otra forma no ocurriría. }\end{array}$ \\
\hline
\end{tabular}

Fuente: AA1000, 2011, p. 19

La estructura básica de la AA1000SES se ajusta al ciclo PHVA (Planear - Hacer - Verificar - Actuar) siguiendo tres tareas principales: (1) mapeo de stakeholders; (2) compromiso con stakeholders, y; (3) evaluación de resultados. Si bien el compromiso es el foco de la planeación, la tarea inicial de identificación de stakeholders se realiza a partir de su clasificación mediante unos atributos dados. En todo caso, la definición de estos atributos no configura un modelo de identificación de actores por sí misma, pues ostenta unas categorías de análisis pero no los criterios para su calificación.

\section{Modelos clásicos para la identificación de stakeholders}

\subsection{Modelo de Gardner (1989)}

Gardner (1989) desarrolla la matriz de poder - interés y poder - dinamismo para la caracterización y ponderación de los stakeholders; se trata de una matriz cartesiana en la cual se ubican los actores identificados por la organización, según su percepción.

Ilustración 6. Identificación de los stakeholders según Gardner (1989)

\begin{tabular}{|c|c|c|c|}
\hline \multicolumn{4}{|c|}{ MATRIZ PODER-DINAMISMO } \\
\hline & & \multicolumn{2}{|c|}{ DINAMISMO } \\
\hline & & BAJO & ALTO \\
\hline \multirow{2}{*}{ 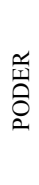 } & BAJO & $\begin{array}{l}\text { POCOS } \\
\text { PROBLEMAS }\end{array}$ & $\begin{array}{l}\text { IMPREDECIBLE } \\
\text { PERO MANEJABLE }\end{array}$ \\
\hline & ALTO & $\begin{array}{l}\text { PODEROSO } \\
\text { PERO } \\
\text { PREDECIBLE }\end{array}$ & $\begin{array}{l}\text { GRANDES PELIGROS } \\
\text { O GRANDES } \\
\text { OPORTUNIDADES }\end{array}$ \\
\hline
\end{tabular}

MATRIZ PODER-INTERÉS

\begin{tabular}{|c|c|c|c|}
\hline & \multicolumn{2}{|c|}{ INTERÉS } \\
\hline & & BAJO & ALTO \\
\hline \multirow{2}{*}{$\begin{array}{l}\frac{\alpha}{1} \\
\text { Oิ } \\
0\end{array}$} & BAJO & $\begin{array}{l}\text { MÍNIMO } \\
\text { ESFUERZO }\end{array}$ & $\begin{array}{l}\text { MANTENER } \\
\text { INFORMADO }\end{array}$ \\
\hline & ALTO & $\begin{array}{l}\text { MANTENER } \\
\text { SATISFECHO }\end{array}$ & $\begin{array}{l}\text { ACTORES } \\
\text { CLAVE }\end{array}$ \\
\hline
\end{tabular}

Fuente: Gardner, 1989. 
La ilustración 6 demuestra cómo el grado de poder de cada actor le permite gestionar sus interacciones con la organización condicionado, a su vez, por el grado de interés o dinamismo que ostente para emprender dichas relaciones.

\subsection{Modelo de Mitchell, Agle y Wood (1997)}

Mitchell, Agle y Wood (1997) explican que los stakeholders son actores (internos o externos) que afectan o son afectados por los objetivos o resultados de una organización dada, en diferentes grados, en la medida en que poseen entre uno y tres atributos básicos: poder, legitimidad y urgencia. Actores que no presentan al menos uno de estos atributos (no afectan los resultados y/o no son afectados por los resultados), no son stakeholders.

Ilustración 7. Identificación de los stakeholders según Mitchell, Agle y Wood (1997)

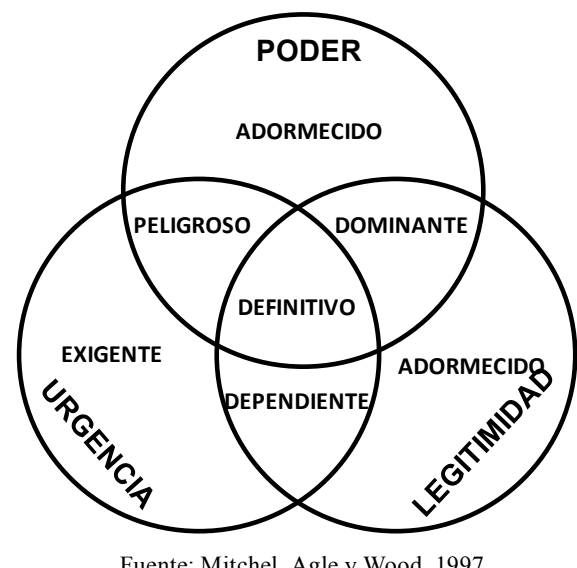

La valoración de este modelo es clara en palabras de Falcao y Fontes (1999):

“(...) este concepto (el modelo de Mitchell, Agle \& Wood, 1997) tiene, por lo menos, tres ventajas sobre los demás: a) es político, pues considera la organización (obviamente, sus propósitos racionalmente definidos) como una resultante (pacto) de intereses conflictivos y desiguales; b) es operacionalizable, pues permite la identificación de stakeholders en la medida en que se pueda evaluar el quantum de poder, legitimidad y urgencia que ciertos actores tienen en determinados tiempo y espacio; y c) es dinámico, no determinístico, pues considera la resultante de intereses en conflicto como móvil en el espacio-tiempo social, de acuerdo con la praxis de los actores." (Falcao y Fontes,1999, p. 9) 
El modelo de Mitchel, Agle y Wood (1997) es en esencia, conceptual, pues los autores no postularon los métodos para su implementación. Con sustento en este modelo, Falcao y Fontes (1999) desarrollan una metodología para la priorización de actores, resultando en un índice de predominancia que resulta de la ponderación de los grados de poder, legitimidad y urgencia de cada actor, según una escala de valoración dada. Así, se convierte en una herramienta de valoración cuantitativa de los atributos de poder. En todo caso, no se hallaron ejemplos de la implementación de este modelo en casos reales.

\subsection{Modelo de Savage - Nix - Whitehead \& Blair. (1991)}

Para Savage et al. (1991), los métodos de identificación de stakeholders usualmente enfatizan en los tipos y la magnitud de las amenazas descartando el enorme potencial de la cooperación, lo que anima a los grupos de interés a trabajar juntos. Esto podría denominarse un "mejor escenario posible" en el que una mayor interdependencia no es una debilidad, sino que representa una oportunidad de intercambio sostenible. Así, Savage desarrolla una clasificación de stakeholders, que combina su potencial de amenaza y su potencial de cooperación, clasificación que conduce a una estrategia de manejo de stakeholders procurando que los grupos de interés adversos a los intereses de la organización cambien su percepción y su actitud.

Ilustración 8. Identificación de los stakeholders según Savage et al. (1991).

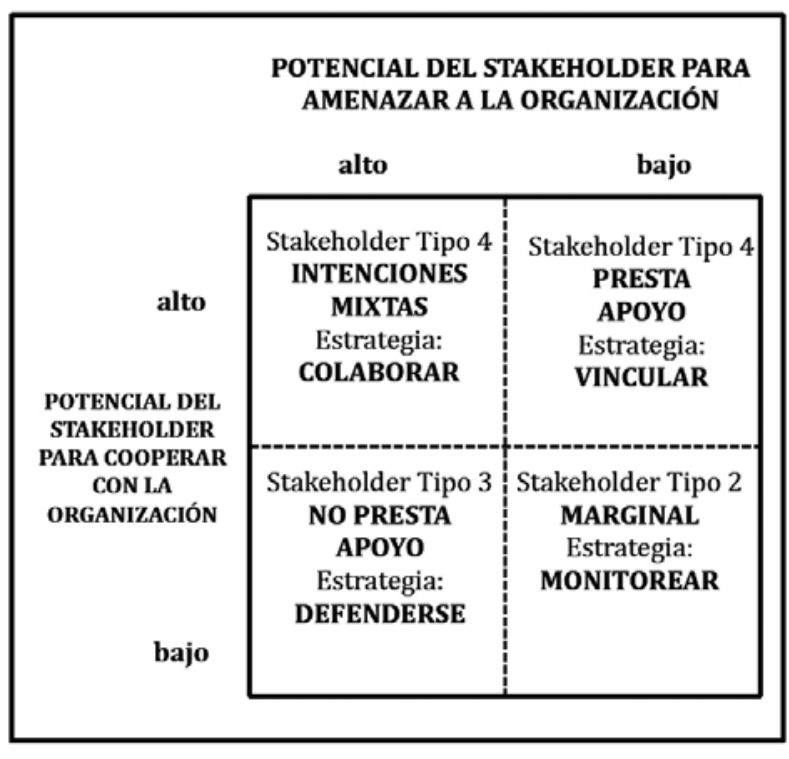

Fuente: Savage et al., 1991, p. 65 
El modelo de Savage et al. (1991) cuenta con amplia aceptación en el ámbito de la dirección. De hecho, Esty y Winston (2009) desarrollan una estructura para el análisis de stakeholders adaptada a partir de la combinación de la calificación de poder-influencia del actor, con su grado de amenaza-cooperación. El ejercicio desemboca en una representación matricial (Ilustraciones 9 y 10):

Ilustración 9. Mapa de Influencia de los actores

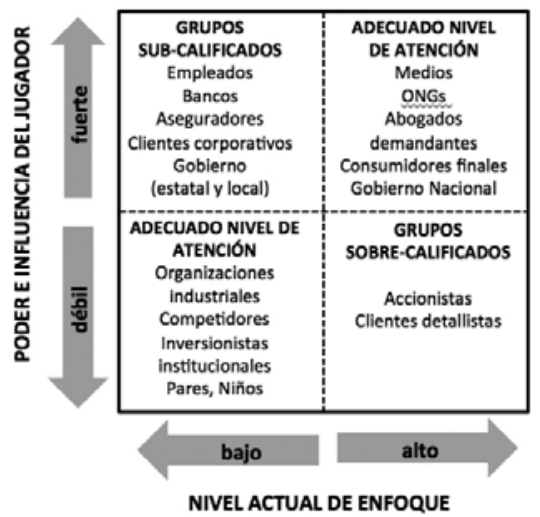

Ilustración 10. Matriz de evaluación del compromiso de stakeholders

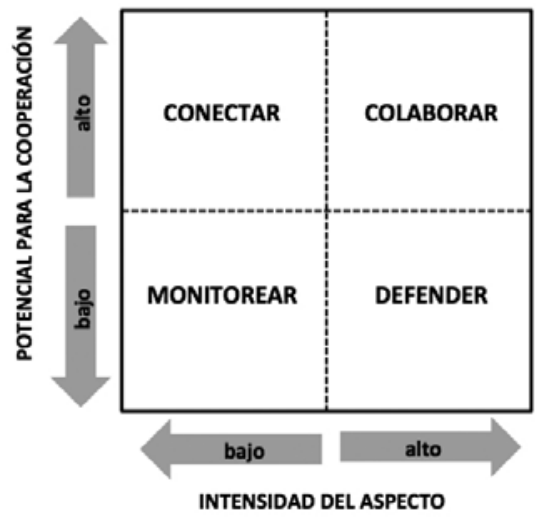

Fuente: Esty y Winston, 2009, pp. 266 y 267 con fundamento en Savage et al.,1991

Si bien las ilustraciones 8,9 y 10 presentan un análisis de stakeholders claro y de fácil comprensión, también exhiben la racionalidad limitada de las representaciones cartesianas. El valor de este modelo radica en las oportunidades que ofrece para entender y explorar el potencial de las relaciones cooperativas con los stakeholders, más que en las virtudes de su representación.

\subsection{Modelo de Cameron - Loureiro \& Rebentisch (2008)}

Cameron et al. (2008) desarrollaron un método completo para la identificación de stakeholders, sus expectativas y las posibles interacciones entre ellos a partir de la caracterización de las necesidades propias de los stakeholders y de las necesidades de la organización respecto de ellos, bajo el supuesto de que se provee un beneficio a un stakeholder a costa de otro lo que, a su vez, requiere la entrega de un valor recíproco. De esta manera, se configura un ciclo de valor (valueloop) integrado por las entradas y salidas entre el stakeholder y el proyecto, que se retroalimentan para el logro de un objetivo dado, en este caso, el éxito del proyecto o la empresa. 
Ilustración 11. Diagrama del ciclo de valor (valueloop) entre dos stakeholders.

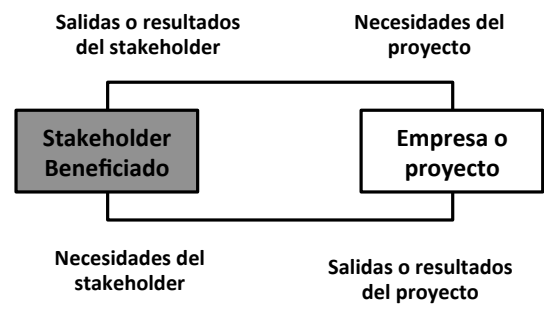

Fuente: Cameron, Seher y Crawley, 2010, p. 2090

El modelo de Cameron se complementa mediante una categorización de los flujos de valor (value flows) en el ciclo de valor (value loop): (1) políticas, (2) dinero, (3) fuerza de trabajo, (4) tecnología, (5) conocimiento, (6) bienes y servicios. De la combinación de los ciclos de valor y sus respectivos flujos entre stakeholders, surge el mapa de identificación de actores. En suma, el ejercicio conduce a una priorización de las necesidades de los stakeholders, no de ellos mismos, y permite la representación de las interacciones dinámicas en un sistema o red interconectada. El modelo, así concebido, fue desplegado para representar las relaciones entre la NASA (Agencia de Aeronáutica y del Espacio de los Estados Unidos de Norteamérica) y sus stakeholders.

Ilustración 12. Modelo de Cameron et al. (2008) aplicado a la NASA

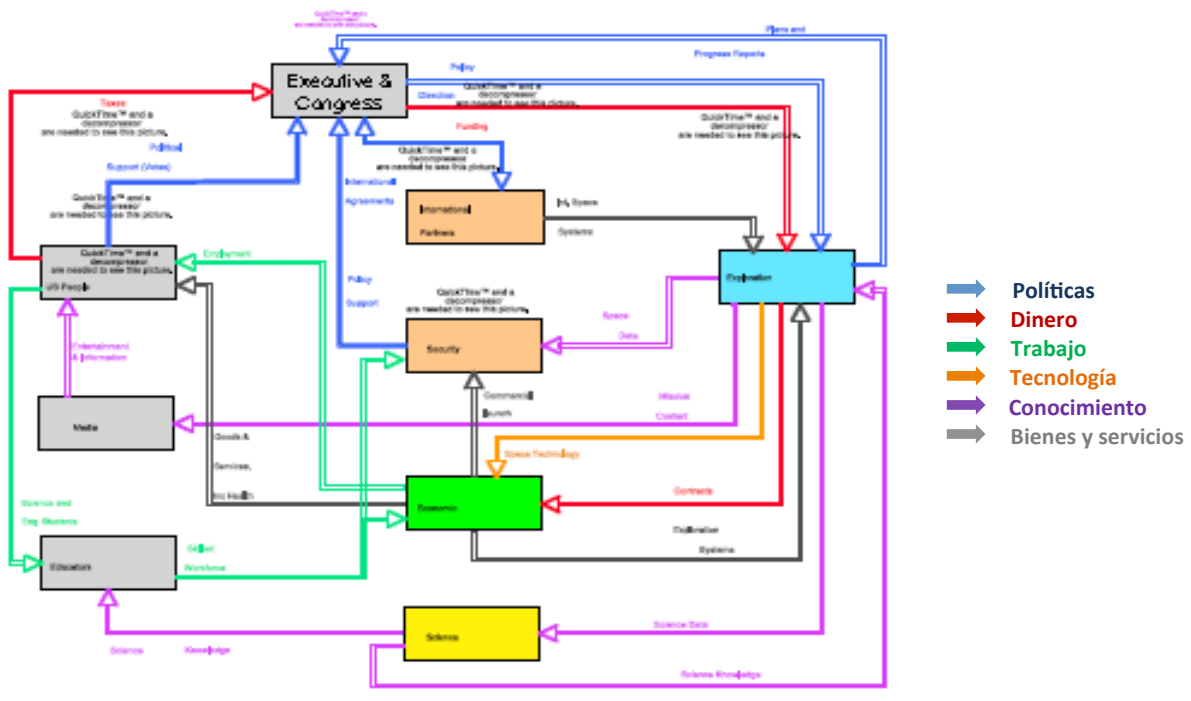

Fuente: Cameron, Loureiro \& Rebentish, 2008, p. 330 


\subsection{Revisión crítica de los modelos clásicos}

Observados los referentes normativos y los modelos clásicos mencionados, es posible identificar las brechas y desafíos del análisis de stakeholders:

Primera: No se cuenta con referentes normativos claros respecto de las consideraciones mínimas que debería tener en cuenta un método de operación, puesto que las guías más avanzadas para el reporte de participación de grupos de interés (GRI y AA1000 series) otorgan un alto grado de libertad para desarrollar la estrategia de stakeholders a la medida de cada organización.

Segunda: Todos los modelos para operacionalizar el análisis de stakeholders desembocan en un ejercicio subjetivo de apreciación de los atributos de poder de cada uno de los grupos de interés. Esto se hace más complejo cuando se realiza un análisis más detallado de las implicaciones de la participación de los stakeholders (Freeman, 2008): (1) Los stakeholders juegan con un abanico de roles (stakeholder roles set), lo que hace que en algunos casos sea apropiado desplegar un análisis de roles. Muchos miembros de un grupo de interés pueden hacer parte de otros grupos, balanceado (o no) sus roles conflictivos y competitivos. De ello pueden derivarse muchas divergencias y expectativas en conflicto frente a la acción de la organización. (2) la interconexión de los stakeholders puede alterar el panorama de amenazas y cooperaciones repentinamente. (3) El análisis de los stakeholders en términos de las percepciones de la organización respecto de su participación no es suficiente. Cuando dichas percepciones no están alineadas, el brillante pensamiento estratégico puede que no funcione. Los stakeholders son humanos y el problema de la congruencia se hace evidente a través de conflictos de valores.

Tercera: La construcción de mapas de stakeholders no es una tarea fácil, en términos de identificar a los grupos específicos y sus participaciones. Los diagramas son demasiado simplificados y los stakeholders aparecen como estáticos pese a que están en continuo cambio, especialmente según el aspecto estratégico en consideración. (Freeman, 2008) De hecho, las representaciones matriciales están restringidas a la comparación entre dos dimensiones o variables, en las que las demás circunstancias se estiman constantes. Esto se aleja de la realidad, puesto que las relaciones entre grupos de interés obedecen a una dinámica constante de cambio e incertidumbre, determinadas además por las interacciones entre stakeholders, que pueden producirse al margen de la empresa. Por otra parte, las representaciones sistémicas permiten incluir una mirada comparada que integra un mayor número de variables, pero pueden resultar confusas para la determinación de objetivos específicos y rutas de acción.

Cuarta: De la misma manera, la construcción de un portafolio adecuado para coordinar los compromisos adquiridos no es una tarea fácil, como lo han demostrado los problemas para crear indicadores claves de desempeño al seguir los patrones cuantitativos y cualitativos del mercado. La tarea se hace aún más dura cuando se enfrentan casos reales de implementación. Si lo deseable es contar con indicadores 
de desempeño e impacto, dicha calificación debería estar integrada al proceso mismo de identificación de actores, para facilitar el seguimiento posterior de los procesos de vinculación y compromiso.

Quinta: Por otra parte, el análisis de stakeholders ha sido aplicado en un sinnúmero de casos, a partir de modelos que pocas veces han tenido en cuenta el desarrollo teórico previo o las consecuencias de su implementación, lo que cuestiona su validez. Al respecto, Perrini \& Tencati (2006) presentan un resumen de las consecuencias de la carencia de modelos confiables y compartidos:

“(1) La existencia de tantos estándares y acrónimos, el desarrollo de tantas propuestas similares y diferentes al mismo tiempo, enfocadas en las expectativas de grandes firmas complican la actitud de las compañías hacia la sostenibilidad, y conllevan una reducción en la adopción e implementación de modelos de gestión más avanzados, en especial en pequeñas y medianas empresas, más que apoyarlas y promoverlas;

(2) Confusión y falta de claridad para los stakeholders de la compañia. Si las firmas no usan una aproximación efectiva y clara en orden amanejar, evaluar y reportar su propio desempeño, los grupos de interés tendrán dificultades en analizar y apreciar los esfuerzos de sostenibilidad de las empresas;

(3) La falta de procesos y métodos reconocidos y compartidos puede recompensar a los gorrones (free riders) que adopten un comportamiento fraudulento y comuniquen resultados falsos, y;

(4) Si las relaciones con los stakeholders son activos esenciales para la creacion sostenible de riqueza, tanto el desempeño de la firma como el desempeño de los stakeholders debería ser medido (...)" (Perrini y Tencati, 2006, p.299)

Sexta: Los desafíos del análisis de actores o stakeholders están lejos de ser resueltos, como resultado de la agitada dinámica de cambio e incertidumbre implícita en todas las relaciones humanas. Sin embargo, precisar las brechas entre los modelos existentes y los retos de su aplicación en la realidad contribuye a la revisión de modelos existentes o al desarrollo de unos nuevos bajo criterios de validez y congruencia, que puedan prestar utilidad práctica en la toma de decisiones. Por otra parte, la variedad de modelos de análisis de stakeholders no debe ser vista por sí misma como una desventaja, por el contrario, los estudios deben apuntar a establecer la validez de un modelo en particular para ciertos casos o contextos en los que su implementación pueda ser exitosa.

\section{Modelos para la identificación de stakeholders de pequeños abastecimientos comunitarios de agua}

El análisis de stakeholders, por sí mismo complejo, implica consideraciones adicionales en el evento de su aplicación en organizaciones de pequeña escala, especialmente cuando debe enfrentarse la realidad de información limitada, escaso desarrollo planeado y restricciones para la acción que acompaña el quehacer cotidiano 
de los pequeños abastecimientos de agua (Godfrey y Howard, 2004). De esta manera, un modelo para la identificación de stakeholders en este tipo de organizaciones debe considerar, a su vez, las capacidades de los responsables de su implementación, los recursos disponibles en el área y los resultados esperados. Es por ello que se recurre a un criterio de validación previa por analogía: para el caso de los pequeños abastecimientos de agua, existen numerosas experiencias documentadas sobre la identificación de stakeholders tras la aplicación de metodologías participativas en el seno de la comunidad. Así, los modelos implementados que se revisan son aquellos que contienen las consideraciones mínimas de los modelos clásicos, y han sido aplicados específicamente al abasto de agua de pequeña escala.

\subsection{Net-Map Tool box (caja de herramientas)}

Usado por primera vez en Ghana por el Comité de la Cuenca del Río Volta, Net-Map Tool box es una metodología para mapear stakeholders que revela las diferencias entre ellos, y crea condiciones para el desarrollo de una visión común, que piensa estratégicamente en oportunidades para la colaboración. El modelo facilita el diálogo acerca de la inclusión y la exclusión, influencia y objetivos, así como enlaces formales o informales y ayuda entre otros propósitos a: (1) comprender redes complejas de políticas (2) determinar quién puede servir como intermediario (3) debatir diversos puntos de vista (3) integrar el pensamiento de redes en la planeación e implementación de proyectos (4) usar las redes para alcanzar el éxito personal. Además, el modelo es susceptible de una construcción participativa y puede ser representado mediante una aplicación digital.

Ilustración 13. Diversas representaciones del modelo Net-Map tool box

Fuente: Net-Map. Website (2012)

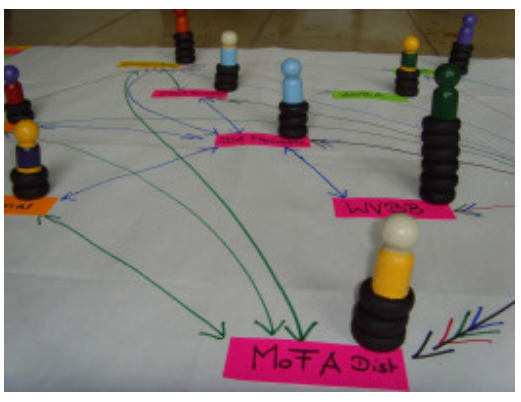

fotografia de un proceso participativo

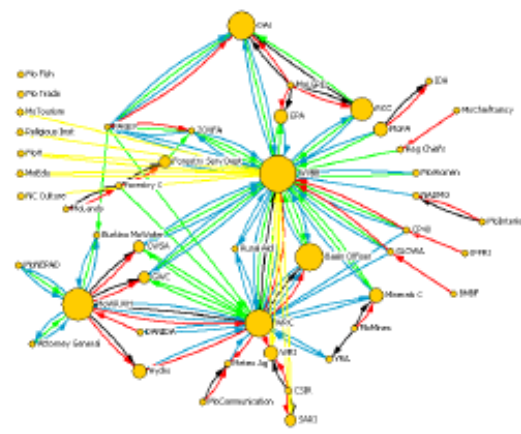

representación computarizada 
La explicación del método en palabras de Shieffer y Peakes (2009), es muy clara:

"Como un tablero de juegos, Net-Map usa figurines para representar diferentes actores implicados en una actividad dada. Entonces se dibujan líneas conectando a dichos actores, usando diferentes colores para representar los tipos de enlace que existen entre ellos, como fuentes de recursos o jerarquías. Después de identificar quiénes están implicados y cómo están entrelazados, el siguiente paso es determinar cómo los diferentes actores pueden influenciar la actividad en cuestión. Para visualizar quien tiene la mayor y menor influencia, los figurines son dispuestos en "torres de influencia" - entre más fuerte la influencia, más alta es la torre. Finalmente, los usuarios mapean los objetivos para cada actor, de manera que todos los objetivos, complementarios o competitivos, sean claros para el grupo". (Shieffer y Peakes, 2009, p. 104)

\subsection{Mapa de actores para organizaciones abastecedoras de agua y saneamiento. DESCOM - FI (FPS, 2009)}

En Bolivia, como parte del Programa "Agua para pequeñas comunidades" del Banco Interamericano de Desarrollo, el Fondo Nacional de Inversión Productiva y Social (FPS) ha formulado un modelo de gestión comunitaria del agua denominado DESCOM-FI (Desarrollo Comunitario y Fortalecimiento Institucional). Este modelo contiene una metodología para desarrollar el mapa de actores para cada comunidad operadora de los servicios de agua y saneamiento. La Ilustración 12 y la Tabla 2 representan la metodología en cuestión.

Ilustración 14. Representación gráfica de mapa de actores - DESCOM FI
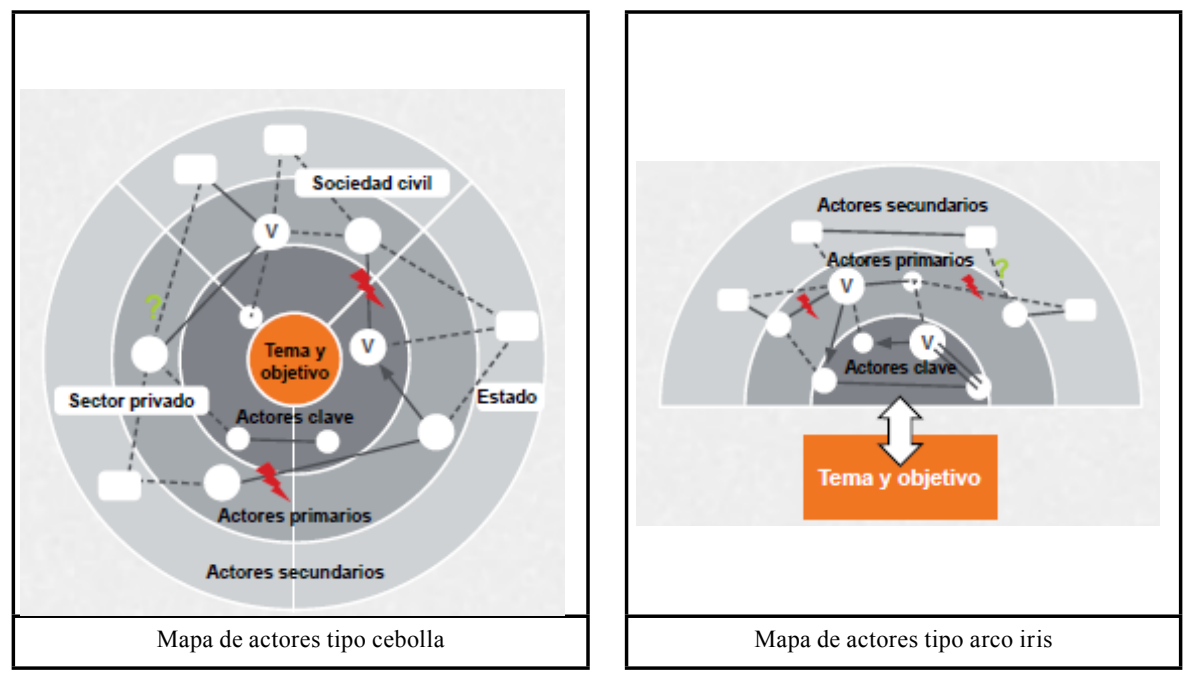
Tabla 2. Pasos para elaborar el mapa de actores en el modelo DESCOM-FI (FPS, 2009)

Paso 1: Identificar a los actores: clave, primarios y secundarios.

Paso 2: Seleccionar la visualización: tipo cebolla o tipo arco iris. (ver Ilustración 12)

Paso 3: Elegir la representación gráfica de los actores: asignar un símbolo para diferenciar a los actores en la Gráfica. Los actores clave y primarios, con un círculo cuyo tamaño guarda relación con su grado de influencia. Si tienen capacidad de veto, se les adiciona una V. Los actores secundarios, con un rectángulo.

Paso 4: Representar las relaciones entre los actores

Simbolizan vínculos estrechos (intercambio de información,

Líneas continuas

la frecuencia de los contactos, la coincidencia de intereses, la coordinación, la confianza mutua)

- ? $_{-} \quad$ Simbolizan los vínculos débiles o informales. Se agrega un

Líneas punteadas signo de interrogación cuando el vínculo no ha sido aclarado.

Líneas dobles

Representan alianzas y cooperaciones reguladas contractual o institucionalmente

Flechas Simbolizan la dirección de los vínculos dominantes

Líneas Representan las tensiones en la vinculación, la contraposición interrumpidas por de intereses y las relaciones conflictivas un relámpago

Líneas transversales Simbolizan los vínculos interrumpidos o destruidos

Paso 5: Colocar los elementos gráficos: hacer que el mapa de actores sea fácil de leer.

Fuente: Adaptado de FPS, 2009, p. 20

El modelo para la identificación y ponderación de actores del DESCOM-FI, es muy interesante por la sistematización y desarrollo gradual del análisis de actores, que parte de una caracterización compleja (grado de poder, importancia y sector al que pertenece, para luego intentar una relación entre estos actores al representarlos bajo una alternativa de visualización que permite representar todas las variables bajo las cuales se desarrolló la caracterización previa. 
De este modelo, no se comparte la clasificación entre actores primarios y secundarios, ya que esta calificación es muy subjetiva y depende de la percepción de un intérprete en un momento determinado. A manera de ejemplo, un actor secundario puede pasar al rol de actor primario bajo circunstancias imprevistas, como podría suceder con el responsable de la atención de emergencias y desastres ante un evento de inundación y tal diferenciación modificaría todo el análisis y su visualización, haciendo muy inestable el mapa de actores logrado bajo consenso de la comunidad. Por el contrario, son muy interesantes las diferencias planteadas entre sectores (privado, público, sociedad civil) ya que estas sí son características permanentes de los actores y plantean la necesidad de perfeccionar las interacciones entre estos sectores, así como las representaciones de las relaciones entre los actores a través de líneas, pues evidencian las posibilidades de mejoramiento.

\subsection{Modelo de mapa de actores para pequeños abastos de agua, Bernal y Rivas, 2007}

La Figura 13 fue creada al combinar los referentes teóricos expuestos en el Aparte 3.4.2., especialmente el modelo para el análisis de actores en comunidades campesinas (ICRA s/f; FAO, 2001) y el modelo para el mapeo de actores DESCOMFI - Bolivia (FPS, 2009).

Ilustración 15. Modelo para la construcción de mapas de actores de pequeños abastecimientos de agua (Bernal y Rivas)

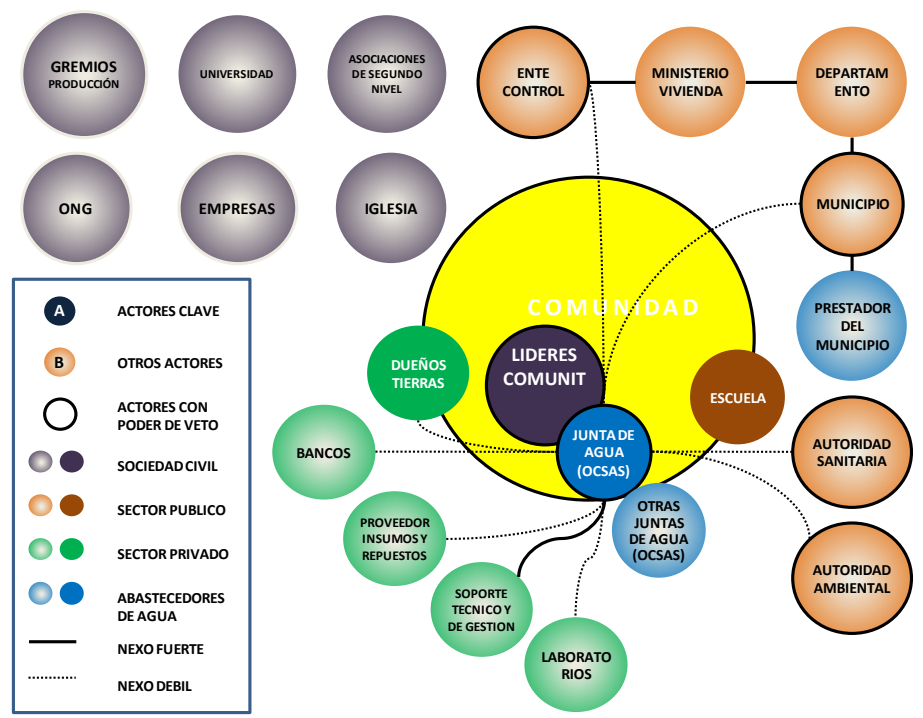

Fuente: Elaboración propia 
En principio, se emplea el diagrama de Venn para identificar a la comunidad como centro del análisis y al acueducto comunitario o Junta de Agua como un grupo de interés. Los actores se clasifican como actores clave, que son los que participan activamente e influyen permanentemente en la gestión del agua, y por ello se representan con colores sólidos y letras blancas. Los otros actores que cuentan con poder e influencia relativos o discontinuos sobre la gestión del agua se representan con colores degradados. Si el actor, tiene poder de veto (puede determinar el cese de operaciones del acueducto comunitario), se encierra en un círculo negro. La sociedad civil, las instituciones públicas, los agentes privados y los abastecedores de agua, se identifican con colores diferentes. Por último, las líneas punteadas de conexión representan los nexos débiles entre actores, y las líneas continuas representan los nexos fuertes.

Esta representación gráfica se formula para permitir la identificación de actores en cada caso específico, con el fin de que contenga la mayor cantidad de información posible en un ambiente gráfico de fácil comprensión y que permita recaudar diferentes percepciones sobre la interacción entre stakeholders. Los mapas de actores suelen hacerse en campo discutida esta representación con los líderes entrevistados y deben plasmarse en un lenguaje sencillo y visual. En suma, el modelo puede ser aplicado a cualquier abastecimiento comunitario de agua y puede ser comprendido y discutido por campesinos, y no se requiere contar con computadores para elaborarlo, pues también pueden recortarse los círculos y hacer un cartel para crear la gráfica en un entorno participativo

Vale anotar que algunos de los actores identificados en la Figura 13 son stakeholders permanentes de cualquier acueducto comunitario: Las autoridades sanitarias y ambientales, el municipio y los entes de control, deben permanecer en contacto con los acueductos comunitarios, en virtud legal. En casi todas las localidades rurales hay al menos una escuela. En este caso, se debe aprovechar el mapa de actores para establecer la naturaleza de las relaciones con estos entes. ¿Se perciben alejados de la comunidad? ¿Se tienen en cuenta sólo porque tienen poder de veto pero no influyen en las decisiones? ¿Los nexos con estos actores del sector público son débiles o fuertes?

Los operadores de agua siempre están y hacen parte de la cuenca hídrica. Siempre habrá otros acueductos comunitarios vecinos y al menos un operador en el Municipio más cercano. Algunos representantes del sector privado están siempre presentes, como son los proveedores de equipos y repuestos, los contadores y personal de apoyo administrativo, los laboratorios y los bancos. Otros pueden estar o no presentes, como son los gremios de producción o las empresas. A su vez, algunos representantes de la sociedad civil están siempre presentes, como son los líderes comunitarios o la iglesia. Otros, pueden participar sólo en algunos casos, como las universidades, las organizaciones no gubernamentales $(\mathrm{ONG})$ o las asociaciones de acueductos comunitarios. 
El objetivo del mapa de actores, en un ambiente de co-gestión, debe dar lugar a una propuesta de arreglos institucionales necesarios para mejorar la interacción de una comunidad y su Junta de agua con sus stakeholders, lo que tiene como finalidad el desarrollo de la estrategia de compromisos compartidos. Por ello en el modelo resultado de esta investigación, se incluyen algunos stakeholders sobre los que puede recaer el análisis, con el fin de explorar oportunidades de inclusión y mejoramiento de relaciones en el escenario local.

\section{Conclusiones}

La teoría de stakeholders configura una estructura explicativa, estratégica, normativa y política para el desempeño de las organizaciones, en un contexto de arreglos institucionales determinado por la interacción entre los grupos de interés en razón de sus atributos. La discusión de esta teoría bajo el enfoque neo institucional conduce a una ampliación de los espacios de racionalidad administrativa que requieren, a su vez, una mayor comprensión de otras dimensiones explicativas, como la dinámica de sistemas o la teoría de la complejidad.

Los distintos modelos descritos comparten el interés por estudiar dos grandes variables que son la cooperación de los actores y su capacidad de amenazar o afectar negativamente a la organización. Las acciones que una organización puede tomar son la Defensa, el Monitoreo, y la Vinculación o la Colaboración.

Estos modelos comparten la lógica de analizar la complejidad inherente a las interacciones humanas y, a su vez, facilitan los procesos de toma de decisiones sobre las acciones que se deben tomar.

La originalidad de este trabajo consiste en que, por lo general, los modelos suelen ser aplicados a grandes organizaciones o a instituciones de gobiernos. En nuestra caso proponemos su uso las localidades rurales y los pequeños abastecimientos de agua que suelen tener complejas interacciones que han sido escasamente abordados en la literatura sobre teoría de la organización.

\section{REFERENCIAS}

AA1000 Stakeholder Engagement Standard (2011) Pre-Publication Versión. Disponible en: www.linkedin.com/groups/AA1000SES-2011-4106960. Consultado el 23 -9- 2012.

Barrau, É. y Frenoux, C. (2010) Services d'eau potable dans les pays du Sud promouvoiretac compagner l'innovation institutionnelle. En Cooperér aujourd'hui No. 68 - Septiembre 2010. Extraído el 24 de octubre de 2010 desde www.gret.org 
Bernal, A. Rivas, L y Peña, P. (2012) Propuesta de un Modelo de Co-gestión para los Pequeños Abastos Comunitarios de Agua en Colombia. Revista Estudios Latinoamericanos. En revisión.

Cameron, B., Crawley, E., Loureiro, G., Rebentisch, E. (2008) Value flow mapping: Using networks to inform stakeholder analysis. Acta Astronáutica 62324 $-333$.

Cameron, B., Seher, T. y Crawley, E. (2011) Goals for space exploration based on stakeholder value network considerations. Acta Astronautica Volume 68, Issues 11-12, June-July 2011, Pages 2088-2097

Donaldson, T y Preston, L.(1995)The stakeholder theory of the corporation: concepts, evidence and implications. Academy of Management Review 20:65-92.

Driscoll, K y Starik, M. (2004) The primordial stakeholder; Advancing the conceptual consideration of stakeholder status for the natural environment. Journal of Business Ethics, Vol. 49 No. 1. (Jan. 2004) pp. 55-73

Esty, D. and Winston, A. (2009): Green to Gold: How Smart Companies Use Environmental Strategy to Innovate, Create Value, and Build Competitive Advantage. Chichester, UK. Wiley.

Falcao y Fontes, (1999) “En quién se pone el foco? Identificando “stakeholders” para la formulación de la misión organizacional" Revista del CLAD Reforma y Democracia No. 15, Oct.- 1999, Caracas.

Fondo Nacional de Inversión Productiva y Social - FPS. (2009) Desarrollo Comunitario y Fortalecimiento Institucional - Instrumento de aplicación: (DESCOMFI). La Paz, Bolivia. Extraído el 15 de septiembre de 2010 desde: www.propac.org

Freeman, R. (1984). Strategic Management: A Stakeholder Approach. Pitman Publishing: Boston, MA.

Freeman, R., Harrison, J. y Wicks, A. (2007): Managing for Stakeholders: Survival, Reputation, and Success. Yale University Press.

Freeman, R., Harrison, J., Wicks, A., Parmar, B., De Colle, S. (2010). Stakeholder theory: The state of the art. Cambridge: Cambridge University Press.

Gardner, J., Rachlin, R., Sweeny, H. and Richards, A. (1989), Handbook of Strategic Planning. R\&D Management, 19: 201. doi: 10.1111/j.1467-9310.1989. tb00639.x

GRI, 2011. Guía para la elaboración de las memorias de sostenibilidad. Disponible en https://www.globalreporting.org/resourcelibrary/Spanish-G3.1-Complete.pdf. Consultado el 23-9-2012.

Kochan, T. y Rubinstein, S., Toward a Stakeholder Theory of the Firm: The Saturn Partnership, Organization Science 11 (4) (2000) 367-386. 
Mitchell, R.K.; Agle, B.R.; Wood, D.J. (1997) Toward a Theory of Stakeholder Identification and Salience: Defining the Principle of who and what really Counts. The Academy of Management Review, 22(4), pp. 853-886.

Net-Map.(2012) Website.http://netmap.wordpress.com/Consultado el 23-9-2012

Perrini, F. y Tencati, A. (2006) Sustainability and stakeholder management: the need for new corporate performande Evaluation and Reporting Systems. En: Business Strategy and the Environment. 15. 296-308.

Pidd, M. (2010) Why modelling and models matter. In: Journal of the Operational Research Society. 61, 1, p. 14-24. Palgrave, MacMillan. London.

Sachs, S. y Rühli, E. Stakeholders Matter: A New Paradigm for Strategy in Society. 0Cambridge University Press, 15 Sep 2011 - Business \& Economics - 296 pages

Savage, G. T., Nix, T. W., Whitehead, C. J., \& Blair, J. D. (1991).Strategies for assessing and managing organizational stakeholders. Academy of Management Executive, 5(2), 61-75.

Schiffer E, and Peakes, J. An innovative approach to building stronger coalitions: Net-Map Toolbox. Development in Practice, Vol. 19, No. 1 (Feb., 2009), pp. 103-105.

Wheeler, D.ySillanpää, M. (1997) The Stakeholder corporation. A blueprint for maximising stakeholder value. London, Pitman Publishing.

WHO (2012a): Water safety planning for small community water supplies: stepby-step risk management guidance for drinking-water supplies in small communities. http://www.who.int/water_sanitation_health/publications/2012/water_supplies/en/ index.htm (Accessed: 2/10/2012) 